\title{
| ARTIGO 1
}

\section{O SIGNIFICADO DA INTERNAÇÃO PSIQUIÁTRICA PARA PACIENTES COM ESQUIZOFRENIA}

Jaqueline Ramires Ipuchimal, Ezequiel Teixeira Andreotti², Jacó Fernando Schneider ${ }^{3}$

Objetivo: compreender o significado da internação psiquiátrica para os pacientes com esquizofrenia. Metodologia: pesquisa qualitativa de natureza fenomenológica pautada no referencial teórico de Maurice Merleau-Ponty, realizada com 10 pacientes com diagnóstico de esquizofrenia, na Unidade de Internação psiquiátrica do Hospital de Clínicas de Porto Alegre (HCPA), no período de junho a agosto de 2016, na cidade de Porto Alegre/RS. Foram realizadas entrevistas semiestruturadas cujas informações foram submetidas à análise fenomenológica. Resultados: emergiram seis unidades temáticas. Conclusão: a partir das colocações de Merleau-Ponty, foi possivel compreender de que forma o paciente com esquizofrenia observa seus avanços ou retrocessos durante o tratamento. O presente estudo torna-se uma ferramenta que possibilita o aprimoramento do processo de atenção ao paciente com esquizofrenia, fazendo com que os cuidados destinados a ele se tornem diferenciados e focados em suas necessidades.

Descritores: Saúde Mental; Esquizofrenia; Enfermagem.

\section{THE MEANING OF PSYCHIATRIC HOSPITALIZATION FOR PATIENTS WITH SCHIZOPHRENIA}

Objective: This paper aims to understand the meaning of psychiatric hospitalization for patients with schizophrenia. Methodology: It is a qualitative research with a phenomenological approach based on the theoretical reference of Maurice Merleau-Ponty, and carried out at the Psychiatric Hospitalization Unit of the Hospital de Clinicas de Porto Alegre (HCPA), Porto Alegre/RS. Results: Ten patients with the diagnosis of schizophrenia were interviewed, and the data submitted to phenomenological analysis from which six thematic units emerged. Conclusion: Based on Merleau-Ponty's findings, it was possible to understand how patients with the diagnose of schizophrenia see their improvements or deterioration during treatment. This study should be used as a tool to allow improvement of the care delivered to patients with schizophrenia, making it more patient-focused and unique.

Descritores: Mental health; Schizophrenia; Nursing.

\section{EL SIGNIFICADO DE LA INTERNA PSIQUIÁTRICA PARA LOS PACIENTES CON ESQUIZOFRENIA}

Objetivo: comprender el significado de la internación psiquiátrica para los pacientes con esquizofrenia. Metodología: investigación cualitativa de naturaleza fenomenológica pautada en el referencial teórico de Maurice Merleau-Ponty. Realizado en la Unidad de Internación psiquiátrica del Hospital de Clínicas de Porto Alegre (HCPA), Porto Alegre / RS. Resultados: fueron entrevistados 10 pacientes con diagnóstico de esquizofrenia, cuya información fue sometida al análisis fenomenológico y emergieron seis unidades temáticas. Conclusión: a partir de las colocaciones de Merleau-Ponty, fue posible comprender de qué forma el paciente con esquizofrenia observa sus avances o retrocesos durante el tratamiento. El presente estudio se convierte en una herramienta que posibilita el perfeccionamiento del proceso de atención al paciente con esquizofrenia, haciendo que los cuidados destinados a él se vuelvan diferenciados y enfocados en sus necesidades.

Descritores: Salud mental; Esquizofrenia; Enfermería.

Complexo Hospitalar da Irmandade Santa Casa de Misericórdia de Porto Alegre/RS

Universidade Federal de Ciências da Saúde de Porto Alegre - UFCSPA/RS.

Universidade Federal do Rio Grande do Sul-UFRGS/RS

Autor correspondente: Ezequiel Teixeira Andreotti. E-mail: andryotti@yahoo.com.br 


\section{INTRODUÇÃO}

Em 1990 foi promulgada a "Declaração de Caracas", em que os países da América Latina se prontificaram a reestruturar a assistência psiquiátrica. Em 2005 a mesma "Declaração de Caracas" foi retomada a fim de avaliar os resultados obtidos desde 1990, reafirmando a necessidade de construção de serviços alternativos aos hospitais psiquiátricos ${ }^{(1)}$.

Como serviços alternativos de assistência psiquiátrica, tem-se: rede de atenção à saúde mental, Centro de Atenção Psicossocial (CAPS), oficinas terapêuticas, residenciais terapêuticos e leitos psiquiátricos em hospitais gerais ${ }^{(2)}$.

Os leitos psiquiátricos existentes nos hospitais gerais são utilizados quando há o esgotamento de recursos extrahospitalares e também, pela necessidade que surge, devido ao quadro clínico do paciente(3). Dessa forma, a internação psiquiátrica, dentro do ambiente dos hospitais gerais, segue sendo um recurso terapêutico indispensável para pacientes com diversas patologias, principalmente as mais graves(4)

Destaca-se a relevância do atendimento deste público alvo nos serviços de saúde, tendo em visto o seu crescimento, cerca de $30 \%$ dos pacientes que procuram o clinico geral é por consequência de algum transtorno mental(5).

Os objetivos da internação psiquiátrica estão centrados na estabilização do paciente, com o intuito de minimizar riscos por meio do levantamento de necessidades psicossociais, ajuste medicamentoso, bem como da reinserção social do paciente em seu meio(b).

Entretanto, é essencial a capacitação dos trabalhadores de saúde e serviços assistenciais, para que possuam manejo adequado e uma visão holística ao assistir este tipo de paciente ${ }^{(7)}$.

No entanto, essa transferência legal de responsabilidade atribuindo novas e especificas demandas para profissionais e serviços também deve ser acompanhada de preparação e capacitação do espaço assistencial, para que a atenção se torne resolutiva e integral, uma vez que a atuação frente aos sujeitos em sofrimento psíquico requer competências particulares.

Dentre os pacientes que, em algumas situações, podem ter indicação de internação psiquiátrica, estão os pacientes com diagnóstico de esquizofrenia.

A esquizofrenia é uma psicose crônica, sem causa conhecida, com um conjunto de sinais e sintomas semelhantes e sobrepostos. Ela tem origem multifatorial, ou seja, fatores genéticos e ambientais estão conectados e associados, aumentando assim, o risco para o desenvolvimento da doença ${ }^{(8)}$. É uma sindrome complexa, sendo considerada por alguns autores como o transtorno psiquiátrico mais grave, podendo gerar prejuízos na vida dos pacientes e de seus familiares, geralmente causando impactos significativos nos seus $\operatorname{cotidianos}^{(9)}$.
A dúvida sobre o conhecimento a respeito do próprio estado de saúde, bem como a compreensão ou não dos motivos que levaram à internação (mesmo após inúmeras internações) desses pacientes sempre foram questionamentos presentes durante o período de prática assistencial dos autores deste artigo.

Apesar dos serviços substitutivos estarem em processo de implantação, ainda há desafios na atenção à Saúde Mental por conta da complexidade da desconstrução do modelo de atenção. Isso inclui mudanças no saber/fazer dos profissionais de saúde. Em busca de um novo olhar, se faz necessário o conhecimento do significado da internação psiquiátrica para pacientes em sofrimento psíquico especificamente, neste trabalho, para os pacientes com esquizofrenia(10)

Frente a isso, este artigo tem como objetivo compreender o significado da internação psiquiátrica para os pacientes com esquizofrenia, tendo em vista que esta percepção é sentida pelo corpo, estando a atitude corpórea do ser humano relacionada ao que o sujeito vive, em que traz consigo diferentes olhares sobre o mundo(11).

\section{METODOLOGIA}

\section{Tipo de estudo}

O presente estudo é uma pesquisa qualitativa de natureza fenomenológica pautada no referencial teórico de Maurice Merleau-Ponty $\left.{ }^{(12}\right)$.

\section{Participantes da pesquisa}

Para participar desta pesquisa foram selecionados 10 pacientes com diagnóstico de esquizofrenia, na Unidade de Internação psiquiátrica do Hospital de Clínicas de Porto Alegre (HCPA), de forma intencional, sendo escolhidos em conjunto com o serviço de saúde, considerando-se a disponibilidade dos sujeitos em participar da investigação. Saliento que todas as entrevistas realizadas na coleta de informações foram utilizadas na análise.

Os critérios de inclusão dos sujeitos do estudo foram: ser maior de 18 anos; ter diagnóstico clínico de esquizofrenia; estar internado há no mínimo uma semana; não apresentar déficit cognitivo e ter condições de estabelecer diálogo.

\section{Local do estudo}

O local escolhido para a realização da pesquisa foi a Unidade de Internação psiquiátrica do Hospital de Clínicas de Porto Alegre (HCPA). 


\section{Coleta dos dados}

A coleta de informações se deu na Unidade de Internação Psiquiátrica do HCPA por meio de entrevista (semiestruturada) fenomenológica no período de junhoagosto de 2016, foi escolhido um local com privacidade, previamente solicitado junto à equipe de enfermagem. Após a assinatura do Termo de Consentimento Livre e Esclarecido (TCLE), as entrevistas foram gravadas em áudio e então, posteriormente transcritas por mim na forma de texto na íntegra, apreendendo-se a comunicação verbal dos entrevistados com intuito de aproximarme ao fenômeno a ser investigado.

Os depoimentos foram então identificados por letras "P" e números seqüenciais ( $\mathrm{Pl}, \mathrm{P} 2, \mathrm{P} 3 \ldots)$, preservando o anonimato dos sujeitos. Houve o cuidado de conduzir a entrevista fenomenológica, ouvindo os participantes sem senso crítico de julgamento e interagindo com os mesmos numa abordagem compreensiva.

Por meio do fenômeno situado, convergências foram buscadas nas unidades de significado, construindo as unidades temáticas. Esse exercício proporcionou uma aproximação do significado da internação psiquiátrica para os pacientes com esquizofrenia para se chegar à essência do fenômeno estudado.

\section{Procedimentos de análise dos dados}

A partir das unidades temáticas, por meio da análise compreensiva dos discursos em Merleau-Ponty, o pesquisador buscou compreender como o esquizofrênico, enquanto participante do estudo, pensa e compreende a internação psiquiátrica, sendo essa a estrutura e essência do fenômeno a ser estudado.

A Fenomenologia é o estudo das essências, onde os problemas se resumem em definir essências dentre as quais estão: a essência da percepção e a essência da concepção. É uma filosofia que integra as essências na existência e não acredita que haja outra forma de compreender o homem e o mundo, a não ser pela facticidade. Assim, há o relato do tempo e espaço do mundo vivido $^{(13)}$.

Assim, neste estudo, o que orientou a atenção para o que se investigou foi a compreensão do viver, através da fenomenologia. Essa permitiu compreender a percepção dos pacientes com esquizofrenia em relação à internação psiquiátrica.

Ao caracterizar esses pacientes, temos que a idade variou de 18 a 60 anos, sem predomínio de faixa etária; $80 \%$ eram do sexo masculino, $50 \%$ tem o Segundo Grau completo, seguidos por $30 \%$ com Primeiro Grau incompleto e $20 \%$ com Primeiro Grau completo. O estado civil dos pacientes aparece com predominância de $90 \%$ solteiros e 10\% divorciados. Já a ocupação variou entre: estudante, aposentado, do lar, mecânico, pintor, assistente de loja, metalúrgico; sendo que 30\% dos entrevistados conta com o Benefício de Prestação Continuada de Assistência Social (BPC).

A entrevista fenomenológica é uma maneira acessível para o sujeito penetrar a verdade mesma de seu existir, sem qualquer falseamento ou deslize, sem qualquer preconceito ou impostura, sendo esta entrevista uma experiência de compreensão, e não uma intervenção.

A fim de compreender o significado da internação psiquiátrica para os pacientes com esquizofrenia, as informações obtidas foram submetidas à análise fenomenológica em consonância com o referencial da fenomelogia da percepção de Maurice Merleau-Ponty. Para a análise das descrições seguiram-se os momentos metodológicos utilizados por Giorgi(14)

Assim, para a análise das descrições foi seguido dos momentos metodológicos, que apresenta quatro passos como procedimento de uma análise fenomenológica:

1. Leitura de cada descrição transcrita na íntegra, com o intuito de dar um sentido geral;

2. Quando o sentido do todo é compreendido, haverá o retorno ao começo, com uma nova leitura com o objetivo específico de discriminar as unidades de significado, com o foco no fenômeno, no caso específico dessa pesquisa, centrado no significado da internação psiquiátrica para os pacientes com esquizofrenia. O sentido geral compreendido depois da leitura do texto não é interrogado nem explicitado em nenhum sentido, servindo de base para o próximo passo, a descrição das unidades de significado;

3. Ao delinear as unidades de significado, as mesmas são explicitadas, expressando o entendimento contido nelas. As unidades de significado revelam a verdade do fenômeno pesquisado, surgindo como consequência da análise, que são descriminações percebidas dentro da descrição da matéria obtida ao se assumir uma postura fenomenológica para com a descrição concreta.

4. Ao final, as unidades de significado serão sintetizadas e transformadas em uma declaração consciente a respeito da experiência demonstrada nos depoimentos, usualmente nominado como estrutura do fenômeno situado.

Por fim, houve a elaboração de uma sintese das unidades significativas, a fim de verificar as convergências 
passiveis de ocorrer entre os participantes com diagnóstico de esquizofrenia analisados em uma mesma categoria de unidades significativas.

\section{Procedimentos éticos}

A pesquisa envolveu seres humanos, o que preconiza conformidade com a Resolução no 466/2012(15), sendo aprovada sob o parecer 1.458 .849 de 21 de março de 2016 . Ressalta-se que também foi obtida formalmente a anuência dos participantes quanto à participação na pesquisa.

\section{RESULTADOS}

\section{Unidades temáticas e estrutura do fenômeno situado}

Ocorreu uma primeira aproximação com o significado que o paciente com esquizofrenia atribui para a internação psiquiátrica, com o intuito de possibilitar a expressão da essência do fenômeno situado.

O fenômeno percebido é vivido pelos pacientes com esquizofrenia no momento em que estão na internação psiquiátrica. Dos depoimentos coletados, emergiram seis unidades temáticas que serão citadas a seguir.

Para os pacientes com esquizofrenia, a internação psiquiátrica significa possiveis melhorias na qualidade de vida

Os pacientes com esquizofrenia, quando internados, depositam esperança na internação psiquiátrica quando atribuem a esta o sentido de que ela pode vir a promover uma mudança positiva em sua estrutura de vida. Relatam que poderão frequentar lugares nunca antes frequentados por associarem essa impossibilidade aos seus níveis de desorganização pré-internação.

"Ela me representa mudar de vida (a internação psiquiátrica), lá fora é uma aqui é outra vida." (Pl)

"É pra eu poder frequentar os lugares que eu não frequentava antes, tipo restaurantes." (P2)

Para os pacientes com esquizofrenia, a internação psiquiátrica significa melhoras no sofrimento psiquico

Ao reconhecerem a presença dos sintomas da esquizofrenia (e o seu sofrimento diante disso) os pacientes acreditam que o significado da internação psiquiátrica é a melhora do seu sofrimento psíquico. Acreditam que o tratamento auxilia na diminuição dos sintomas da doença, bem como do sofrimentos psíquico que ela causa.
"O significado é a minha melhora. Minha melhora... tem muitas coisas que eu tenho que fazer lá fora, que eu tenho que vencer [...] o significado da internação é que eu estou na expectativa de melhoras, de melhorar e passar bem." (P2)

"Significa muito, significa que eu melhore, por que eu faço tratamento de ECT (eletroconvulsoterapia) que é para melhorar a cabeça mesmo." (P3)

Para os pacientes com esquizofrenia, a internação psiquiátrica significa a possibilidade de concretizar planos e desejos no futuro

Nas falas analisadas, foi possivel observar que os pacientes com esquizofrenia atribuem como significado de sua condição de internação a possibilidade de concretizar planos e desejos para o futuro. Reconhecem que ao melhorarem dos sintomas, através de tratamento oferecido pelo serviço, é possivel estabelecer metas para o período pós-alta e tentar realizá-las.

"A melhora pra mim é minha felicidade, meus objetivos. Eu pretendo fazer muitas coisas que não fazia antes. [...] poder fazer muitas coisas que eu não fazia antes" (P2)

"Está tendo um significado de coisas boas, de continuar tomando o remédio, de talvez lá no meu bairro conseguir algum curso próximo, de tocar o barco, de não desistir." (P5)

Para os pacientes com esquizofrenia, a internação psiquiátrica significa terem um cuidado centrado e individualizado

Nesta unidade temática, é possivel compreender que o significado da internação psiquiátrica, para os pacientes com esquizofrenia, tem ligação com a possibilidade de um tratamento centrado e individualizado. O paciente sente que é singular frente a todos os seus problemas e considera que esse cuidado centrado e individualizado é o significado de estar internado.

"Eu acho bom a internação. Eles são bem acolhedores, eles centram a gente. Eles sempre tão ajudando cada vez que a gente faz um ECT, a gente fica mais acolhido por eles, por que eles sempre tratam a gente com muita educação, tratam a gente bem." (P3)

"É um lugar bom, tem os profissionais, são legais, o ambiente é bom." (P4)

Para os pacientes com esquizofrenia, a internação psiquiátrica significa a socialização como um disparador de novos aprendizados

$O$ paciente com esquizofrenia, quando numa internação psiquiátrica, acredita que um dos significados 
que esta traz é o da possibilidade de socialização. Essa socialização vem acompanhada de novas descobertas e aprendizados de atitudes e atividades que esse paciente não tinha e não fazia no seu cotidiano.

"As vezes, é meio dificil por que tem outros paciente, conviver com outros paciente tem problema, quando o paciente está muito agitado... esse é um negócio que a gente vê várias pessoas com problema diferente. Tu vê que tem pessoas que tem o problema maior que o seu." P4

"Indo participar ali na recreação consigo aprender a mudar a rotina lá fora." (P8)

"É estar perto de outras pessoas, fazendo atividade em conjunto, coisas que eu posso fazer lá fora também [...] quero mudar quando eu sair daqui." (P8)

Para os pacientes com esquizofrenia, a internação psiquiátrica significa segurança

Essa unidade é correspondente ao sentido de segurança que o paciente com esquizofrenia dá para a internação psiquiátrica. Ele acredita que dentro da internação é possivel sentir-se seguro visto que o manejo é diferente do que habitualmente está acostumado. Entende que, ao não conseguir conviver com seus sintomas no cotidiado, a internação psiquiátrica pode auxiliar nessa sensação de segurança até a melhora.

“É melhor estar internado, sabia? Por que lá fora tá inseguro o mundo, e a pessoa livre tá arriscando a vida... qualquer coisinha é arriscado lá." (Pl)

"Eu gosto mais da internação, por que em casa eu viveria no cadeado. Eu tentava pular o muro de 3 metros, com vidro em cima; se eu pulasse, eu me rasgava, me cortava, não estou ainda muito bem. Sinto que estou bem aqui internado." (P9).

\section{DISCUSSÃO}

Compreendendo o significado da internação psiquiátrica para os pacientes com esquizofrenia

Nesta etapa da análise, é realizada uma interlocução das idéias dos pacientes com esquizofrenia sobre a internação psiquiátrica com a concepção teórica de Maurice Merleau-Ponty. Frente à questão norteadora: "Qual o significado da internação psiquiátrica para você?", emergiram percepções variadas dos pacientes.

Nas falas dos sujeitos com esquizofrenia podemos observar que sua percepção sobre a internação psiquiátrica emerge de forma a entendê-la como uma "possibilidade de melhorias", tanto na qualidade de vida quanto no sofrimento psíquico, que o levou à internação. $O$ sujeito percebe que há mudanças positivas no período em que está internado e atribui o significado de que seja possível levar essa mudança para seu cotidiano fora da internação.

\begin{abstract}
"Só se compreende a significação motora das cores se elas deixam de ser estados fechados sobre si mesmos ou qualidades indescritiveis oferecidas à constatação de um sujeito pensante, se elas atingem em mim uma certa montagem geral pela qual sou adaptada ao mundo, se elas me convidam a uma nova maneira de avaliar e se, por outro lado, a motricidade deixa de ser a simples consciência de minhas mudanças de lugar presentes ou futuras para tornar-se a função que, a cada momento, estabelece meus padrões de grandeza, a amplitude variável de meu ser no mundo" (12:283).
\end{abstract}

As falas dos pacientes com esquizofrenia também trazem consigo a forma como os mesmos utilizam seu corpo como sinalizador de sua melhora. O sinal é percebido no momento em que o sujeito verbaliza identificar melhora dos sintomas psíquicos durante o tratamento, o que faz com que se sinta motivado a estabelecer metas pós-alta e a tentar realizá-las.

\footnotetext{
"Ser corpo, nós o vimos, é estar atado a um certo mundo, e nosso corpo não está primeiramente no espaço: ele é o espaço. [...] Se ainda se pode falar, na percepção do corpo próprio, de uma interpretação, seria preciso dizer que ele se interpreta a si mesmo" (12:205-8).
}

O significado da internação psiquiátrica para o paciente com esquizofrenia também suscita a questão do cuidado centrado e individualizado e a necessidade que este sujeito verbaliza ter para que isso ocorra. O paciente percebe que é um ser único e singular nos fazendo compreender que, embora o diagnóstico seja o mesmo, sua individualidade é essencial e deve ser preservada e valorizada.

\footnotetext{
"A existência pessoal é intermitente e, quando essa maré reflui, a decisão só pode dar à minha vida uma significação forçada. A fusão entre a alma e o corpo no ato, a sublimação da existência biológica em existência pessoal, do mundo natural em mundo cultural, é tornada ao mesmo tempo possivel e precária pela estrutura temporal de nossa experiência" (12:125).
}

Essa existência humana também pode ser entendida como necessária à socialização. Esta, por sua vez, aparece nas entrevistas dos sujeitos como disparadora de novos aprendizados, novas sensações, novos conhecimentos e acontecimentos.

Merleau-Ponty, diz que é por meio das situações que desacomodam que o sujeito entrega-se à necessidade de utilizar-se de meios que disparem sua desacomodação: 


\begin{abstract}
“Na montanha, um esquizofrênico detém-se diante de uma paisagem. Depois de um momento, ele se sente como que ameaçado. Nasce nele um interesse especial por tudo o que o circunda, como se, do exterior, lhe fosse posta uma questão para a qual ele não pôde encontrar resposta. Repentinamente, a paisagem lhe é arrebatada por uma força estranha. É como se um segundo céu negro sem limites, penetrasse no céu azul da tarde. Esse novo céu é vazio 'fino', invisivel, horrivel. Ora ele se move na paisagem de outono, ora ela também se move. E durante esse periodo, diz o doente, 'uma questão permanente se põe a mim; é como uma ordem crescente de descansar ou de morrer, ou de ir mais adiante" (12:385).
\end{abstract}

As sensações do paciente com esquizofrenia durante a internação psiquiátrica também englobam o sentido de segurança, de cuidado. Nas falas dos pacientes, emergem significados que vieram de sua percepção de mundo, de suas vivências em relação ao "estar em segurança”.

\begin{abstract}
"Quando digo que tenho sentidos e que eles me fazem ter acesso ao mundo, não sou vítima de uma confusão, não misturo o pensamento causal e a reflexão, apenas exprimo esta verdade que se impõe a uma reflexão integral: que sou capaz, por conaturalidade, de encontrar um sentido para certos aspectos do ser, sem que eu mesmo tenha dado a eles por uma operação constituinte" (12:292).
\end{abstract}

O sujeito com esquizofrenia consegue ter diversas percepções de seu ambiente, entendendo a internação para além da melhora dos sintomas psiquiátricos, para além do tratamento, entendendo que pode ser um momento de novos conhecimentos, rumos novos, planos novos. Utiliza-se de seu corpo como sinalizador de suas mudanças, entendendo-se enquanto um ser único.

“[..] ele mesmo percebe, que ele é o sujeito perceptivo e que a percepção, tal como ela vive, desmente tudo o que ele diz da percepção geral. Pois, vista do interior, a percepção não deve nada àquilo que nós sabemos de outro modo sobre o mundo, sobre estimulos tais como a física os descreve e sobre os órgãos dos sentidos tais como a biologia os descreve" (12:279).

A partir das colocações de Merleau-Ponty, referente à Fenomenologia da Percepção, podemos compreender melhor o entendimento que o paciente com esquizofre- nia dá para sua internação psiquiátrica e de que forma ele consegue observar seus avanços ou retrocessos durante o tratamento.

\section{Limitações do estudo}

A limitação deste estudo refere-se à escassez de artigos relacionados aos pacientes com esquizofrenia e foi o que impactou diretamente na elaboração desse artigo visto que a discussão necessita de argumentos para auxiliarem no debate. Além do desafio da pesquisa, por não ser fácil entrevistar um paciente com esquizofrenia, precisando de manejo adequado do profissional da saúde para abordagem eficaz.

Contribuição do estudo para a prática

A contribuição desta pesquisa é de extrema relevância para fomentar mais publicações relacionadas à pacientes com quadro esquizofrênico.

\section{CONCLUSÃO}

A percepção do paciente com esquizofrenia é carregada de conceitos que este adquiriu durante suas internações. É a partir desses conceitos que o paciente se dispõe a perceber de que forma pode levar sua internação, percebe seu corpo, se encontra como ser no mundo e inicia seu reconhecimento.

A pesquisa vem com a possibilidade de inserção de novos modelos de cuidado em saúde mental para os pacientes com esquizofrenia. Também é de extrema relevância que o paciente com esquizofrenia tenha a possibilidade de expor suas percepções em relação à sua condição para que seu tratamento seja potencializado.

O enfermeiro pode instrumentalizar-se para que a terapêutica com relação ao paciente seja adequada, melhor recebida por ele e melhor entendida pelos outros membros da equipe.

\section{Contribuição dos autores}

Concepção e/ou desenho: Jaqueline Ramires; análise e interpretação dos dados, redação do artigo, revisão final, revisão crítica: Jaqueline Ramires Ipuchima, Jacó Fernando Schneider, Ezequiel Teixeira Andreotti. 


\section{REFERÊNCIAS}

1. Ministério da Saúde (BR). Carta de Brasilia Principios Orientadores para o Desenvolvimento da Atenção em Saúde Mental nas Américas. 2005. Available from: http://www.crpsp.org.br/povos/ povos/legislacao/Carta\%20de\%20Bras\%EDlia\%20de\%202005\%20 MS_OPAS_OMS.pdf

2. Hirdes A. A reforma psiquiátrica no Brasil: uma (re) visão. Cien Saude Colet [Internet]. 2009:14(1):297-305. Available from: http://www.scielo.br/scielo.php?script=sci_arttext\&pid=S1413$81232009000100036 \&$ ing=pt\&tlng=pt

3. Pacheco MA, Cataldo Neto A, Menezes F, Krieger CA, Bersano L Gil A. Aspectos do funcionamento de uma unidade de internação psiquiátrica de um hospital geral. Rev Psiquiatr do Rio Gd do Sul [Internet]. 2003;25(suplemento 1):106-14. Available from: http://www.scielo. br/scielo.php?script=sci_arttext\&pid=S0101-81082003000400011\&lng=en\& $n r m=i s o \& t \operatorname{lng}=p t \% 5 \mathrm{Cnhttp}: / / \mathrm{w} w \mathrm{w}$. scielo.br/scielo.php?script=sci_arttextEpid=S0101-81082003000400011Elng=ptEnrm=iso\&tlng=pt

4. Dalgalarrondo P, Botega Neury J, Banzato Cláudio E M. Pacientes que se beneficiam de internação psiquiátrica em hospital geral Rev. Saúde Pública [Internet]. 2003 [cited 2019 Jan 30] :37(5) 629-634. Available from: http://www.scielo.br/scielo.php?script=sci_ arttextEpid=S0034-89102003000500013Elng=en. http://dx.doi. org/10.1590/S0034-89102003000500013.

5. Batista REA, Campanharo CRV. Avaliação da adesão ao tratamento medicamentoso de pacientes com distúrbios psiquiátricos no serviço de emergência. Enferm Foco [Internet]. 2018 [cited 2019 Jan 30]:9(4):8-12. Available from: http://revista.cofen.gov.br/index.php/ enfermagem/article/view/1271/468

6. Cardoso L, Galera SAF. Internação psiquiátrica e a manutenção do tratamento extra-hospitalar. Rev. esc. enferm. USP [Internet]. 2011 [cited 2019 Jan 30] :45(1): 87-94. Available from: http://www.scielo. br/scielo.php?script=sci_arttext\&pid=S0080-62342011000100012\&lng=en. http://dx.doi.org/10.1590/S0080-62342011000100012.

7. Oliveira LC, Rafael F, Soares R, Miranda K, Sousa N. Dificuldades no atendimento às urgências e emergências psiquiátricas no serviço de atendimento móvel de urgência. Enferm Foco [Internet]. 2018 [cited 2019 Jan 30]:9(3):18-22. Available from: http://revista.cofen.gov. br/index.php/enfermagem/article/view/1317/471

8. Silva RC. Esquizofrenia: uma revisão. Psicol USP [Internet]. 2006 [cited 2019 Jan 30];17(4):263-85. Available from http://www.scielo. br/scielo.php?script=sci_arttext\&pid=S0103-65642006000400014Elng=en\&nrm=iso

9. Santana AFFDA, Chianca TCM, Cardoso CS. Qualidade de vida de pacientes com esquizofrenia internados em hospital de custódia. J Bras Psiquiatr [Internet]. 2009 [cited 2019 Jan 30] ;58(3) 187-194. Available from: http://www.scielo.br/scielo.php?script=sci_ arttextEpid=S0047-20852009000300008\&lng=en. http://dx.doi. org/10.1590/S0047-20852009000300008

10. Mielke FB, Kantorski LP, Jardim VM da R, Olschowsky A, Machado MS. O cuidado em saúde mental no CAPS no entendimento dos profissionais. Cien Saude Colet [Internet]. 2009 [cited 2019 Jan 30];14(1):159-164. Available from: http://www.scielo.br/ scielo.php?script=sci_arttextEpid=S1413-81232009000100021\&lng=pt\&tlng=pt

11. Nóbrega TP. Corpo, percepção e conhecimento em Merleau-Ponty. Estud Psicol [Internet]. 2008 [cited 2019 Jan 30];13(2):141-148. Available from: http://pablo.deassis.net.br/wp-content/uploads/06.pdf

12. Maurice MP. Fenomenologia da percepção. Tradução de Carlos Alberto Ribeiro de Moura. 4. ed. São Paulo: Martins Fontes, 2011.

13. Dalva M, Carvalho DB, Martins R. A pesquisa fenomenológica e a enfermagem. Acta Scientiarum [Internet]. 2002 [cited 2019 Jan 30]; 24(3):843-847. Available from: http://periodicos.uem.br/ojs/index. php/ActaSciHealthSci/article/view/2545

14. GIORGI, A. Phenomenology and Psychological Research. Pittsburg: Duquesne University Press, 2003.

15. Conselho Nacional de Saúde. Resolução (BR). Resolução no 466 de 12 de dezembro de 2012. Diretrizes e normas regulamentadoras de pesquisa envolvendo seres humanos. Brasilia, 2012. Available from: http://conselho.saude.gov.br/resolucoes/2012/reso466.pdf 